\title{
Urban Public Transportation Network Planning Method Based on Transit-oriented Strategy
}

\author{
Ming Gu, Bin xu, \\ Transportation and Management College \\ Dalian Maritime University \\ Dalian, China
}

\author{
Yongzhong $\mathrm{Hu}$ \\ Transportation and Management College \\ Dalian Maritime University \\ Dalian, China
}

\begin{abstract}
This article proposed a transit-oriented planning for urban public transport situation. Adjust the existing passenger OD matrix according to the city development plan. Propose an optimization on the adjusted OD matrix to achieve the shortest travel time, direct access to the largest passenger flow target. Heuristic method to optimize the use of public transportation network, according to city traffic conditions and specific feedback on the optimization results are corrected, obtained satisfactory results.
\end{abstract}

Keywords-Transit-oriented, OD Matrix, Heuristic, Feedback and adjustment

\section{INTRODUCTION}

As China's economic development, rapid expansion of urban development, population growth, urban traffic problems become more and more residents living between urban development and the key. The bus traffic in the city's position is self-evident, it has a low cost, high efficiency, service wide and strong stability and many other advantages. A good public transport system will attract more passengers, the maximum possible pressure to ease the urban traffic, Not only that, it can not only passively accept the passenger flow, it also can initiative to promote the passenger flow. So the public transportation network has a certain oriented. But how to guide, and guide the future development of the city used are transit-oriented concerns, is the focus of future research. This article discusses the policy-oriented public transport and the corresponding optimization methods.

\section{TRANSIT ORIENTED}

\section{A. What is Transit Oriented}

Urban public transit network planning based on planning objectives, requirements, time constraints and financial constraints can be divided into the recent planning and long-term planning.

Recent planning is to optimize the adjustment of the existing bus system, Although the short-term benefits and local benefits are obvious, and the investment of human and material resources and little money, but bound by the status of urban transportation more, only to supplement the existing city bus module problems. Planning result can only be an imperfect adaptive bus network.

Long-term planning is the best goal of urban transportation systems, from a global perspective, combined with the city's future development strategy of the characteristics of urban public transport has developed a comprehensive and sustainable development programs.It is dedicated to the city's traffic problems in the future to be a fundamental solution.It is not subject to the constraints of existing urban traffic conditions, it, has greater flexibility, however, human and material resources and capital investment is relatively large. This advanced idea is called transit-oriented strategy (Transit Oriented Development, TOD[8]). TOD was first proposed by Peter Calthorpe in California school. It stressed that public transport and close integration of land use planning, promote the formation of the corridor as a link to public transport, public transport oriented, comprehensive land use groups as nodes of urban layout.

Based on reality, Transit-oriented strategy for urban public transport networks tend to want to reshuffle the cards, plans to build a science city bus system will greatly benefit the future development of the city. However, some large-scale development of the city's public transport network is difficult to implement.

\section{B. Public Transit-oriented strategy}

The object of public transportation network, including station for concentrated stop, line, station three basic elements. They are also called the point, line and plane in transit planning.Public transportation network planning is essentially set by a reasonable point, line, surface between the public transport system layout to maximize the function. It can be divided into adaptive public transportation network planning and oriented public transportation network planning.

Adaptive public transportation network planning thought is: Status quo through restructuring and optimization of transit network layout to achieve a reasonable purpose of route planning transport hub.That first network and then hub.

Oriented public transportation network planning thought is: Laid hub according to the long-term demand for public transport passenger.then laid network on basis of new 
hub.That first hub and then network, anchoring line network through the hub.

Oriented public transportation network planning will be guided to achieve the overall urban transport, urban land use and transport system interaction, and ultimately the city's new layout. Public Transit-oriented planning process is characterized by the planning and foresight, the first blueprint for urban development planning, the establishment of a new bus-oriented set of nodes, and then predict the OD plan will be achieved after the passenger, and then formation of oriented And the predictability of the OD matrix, forming a new transit hub. These are the focus of the public transportation network optimization.

\section{HEURISTIC OPTIMIZATION METHOD BASED ON OD MATRIX AND THE CITY POSSIBLE BUS ROUTE MAP}

\section{A. General public transportation network optimization}

Public transportation network optimization problem belongs to the network optimization problem on the map.It is an extension of TSP problem and quadratic assignment problem.It is a kind of composite type of NP problems.

It's solution has considerable spatial complexity and time complexity. Although some mathematical methods to get the optimal solution in theory, but with the increasing size of the problem will appear the phenomenon of combinatorial explosion, so far, most people use heuristic methods and some new evolutionary algorithm to optimize the complex bus system. Common algorithms are

1)global optimization method

More than the entire line of network objectives classified into a linear function, and put all the variables multiplied by the coefficient of relative effectiveness in meeting the total calculated under the constraint of the consumption charges. If ANBansal [3] proposed the passenger travel time, waiting time based on the cost of income into the assessment, the goal is to meet the constraints so that all passengers under the car and the waiting time and vehicle operating costs and expenses Minimum. The establishment of pure linear optimization methods are often more complex objective function, constraints, too, to solve very complex problems are only suitable for small-scale solution of the status quo and ignores some of the practical factors of public transportation network.

2) One by one into a network layout optimization

Optimization goal for all mathematical models, the model solution is very difficult, now more a wire mesh is the core of performance indicators for the goal, while other indicators will be transformed into constraints to solve. One by one into a network layout optimization method based on one or more core indicators focus on the possible routes, one by one to find the best bus lines, superimposed into a network, and through the Network Assignment or a series of tests to form a network of local modified and improved. The idea is simple and intuitive, easy to implement, in the optimization process in the feedback adjustment of subjective and experiential, is now the main line network adjustment methods.

3) Intelligent search algorithm ant colony algorithm

This type of evolution with intelligent search algorithm is very suitable for solving linear programming problems, but the face of network planning, in particular, a large transit network planning such a complex system, the development is not yet mature ant colony and particle swarm Algorithm to solve this problem show much less, such as the convergence rate is too slow, and prone to premature phenomena; there is the artificial control of the poor. However, these algorithms can be expected broad prospects for development.

4) Improved method for current situation

This approach is fully maintaining the status quo in the reasonable part of the bus lines, and the passenger pre-distribution, low efficiency out of line, and then one by one by remaining OD Method of setting the amount of wiring, the last passenger network through a series of tests to distribution and partial adjustment Lines, to optimize the purpose of transit network. Obviously this method is suitable for adaptation planning public transportation network, but not for oriented strategy.

Now all the optimization problems are NP problems can not solve the local optimal solution to the global optimum convergence of degree. Above algorithm is easy to fall into local optimal solution and the computation is slow, it is difficult to solve for the actual large-scale problems, in this case, the heuristic algorithm as $\mathrm{a}_{*}$ good choice. In the heuristic algorithm we use $C / C \leq \varepsilon$ to measure the algorithm is good or bad, $C$ is the solution of the target, ${ }^{C}$ is the optimal solution of the target, $\varepsilon$ is the heuristic solution and optimal solution on the ratio of the target community at worst case. This heuristic allowed to become an effective control method.

\section{B. Heuristic optimization method based on OD matrix and the city possible bus route map}

1) OD matrix

Before the optimization algorithm described first introduce the concept of OD matrix. Public transportation network optimization methods rely on the status of a city bus statistics, that is OD volume and OD matrix.

OD matrix is the n-order square matrix, $n$ behalf of the total number of traffic nodes, $i$ and $j$, said urban transport nodes, ${ }^{i j}$ Said that from $\mathrm{i}$ to $\mathrm{j}$ of the OD traffic, The last line of each point of the input data that the total passenger flow OD, the last column of data that each point of the total output of passenger flow, the lower right corner of the OD data representation of the total urban passenger throughput.

2) Optimization

First Public Transit-oriented planning of urban land use planning need to create a new public transport nodes in the set and the expected OD matrix. In the new OD matrix is optimized to select the most central city bus a few goals, the laid one by one, superimposed into a network. Test conditions as other secondary target after the online network generated by a certain way to adjust. By studying the survey, 
choose to travel the shortest time to reach the highest at the core of the optimization objectives. Let the total number of nodes in the OD matrix is $\mathrm{n}$, through the road map will be connected to $\mathrm{n}$ nodes $n(n-1) / 2$ unicom may constitute a path. Dijkstra's algorithm with each path between two points in the city to determine the shortest path. Now each path corresponds to the OD traffic matrix in the value of 2 , one for this round of the passenger path, this path is the shortest travel time and the direct path of the highest rates.

Specific method steps are:

a) We retained the first ${ }^{n(n-1) / 2}$ routes to and from the passenger and the largest piece, as the initial route network.

b) And then selected for the initial line net amount of line OD OD from the total amount of nets removed, and the starting point of the end of the line starting from the planning focus on removing the terminal, when a bus from the terminal a number of the The departure of the bus lines, it will thus originating from the terminal car paths number minus 1, get a new set of lines and bus lines from the terminal OD table.

c) According to the repeated factor correction circuit operating time, and if some of the bus lines on the road too much, it will affect the city's public transportation network of the uniform distribution, and other vehicles traveling on the road, so after the establishment of a bus line, as far as possible in the future with the establishment of this rail line duplication, the introduction of the road which has been set up with the bus number of the double-track section coefficient $\mathrm{k}$, on which bus lines run all the time to amendment to the road, the actual running time multiplied by the double-track road as the next coefficient stage of the route of the running time, double-track line coefficient is too large may cause excessive twists and turns, an increase in ridership distance; too small will not achieve the role of double-track control. Repetition factor can refer to the following diagram:

d) The operation of the previous cycle, according to the merits of the order generated by one bus line network or the initial set of initial nets.

\section{3) Inspection and Adjustment:}

Obtained after optimization of the initial route network to be inspected and adjusted. Because in addition to high travel time is short and direct but also to meet other than travel by other requirements, such as load factor to a large line, line of moderate length, the average transfer times less, the line up is good and so on.

1) OD matrix based on the calculation of each row and each column, that is obtained from various points of departure and arrival of the OD volume, then each point of departure and arrival passenger passenger OD this point is derived by adding the total throughput. The maximum throughput of the node as a new transportation hub planned. OD points will be sorted descending throughput, greater value the more important of its traffic status. To each node on the line of traffic throughput of the combined transport and obtained the degree of importance of each line in the order.

2)According to an important degree of nodes and lines in the order of the lines investigated whether the standard of satisfaction,Including non-linear coefficient index line ${ }^{l_{i j} / d_{i j} \leq 1.4},{ }_{i j}$ is the the length between I,j line, ${ }^{d}{ }_{i j}$ is the the Straight-line distance between I,j line.Full line rate index, $\eta=Q_{k} / Q_{\max } \leq 60 \%$,Is the actual carrying capacity and the rated capacity of more than. Average transfer times $\bar{X}=\left(\sum_{n} \sum_{n} T_{i j}^{0}+2 T_{i j}^{1}+3 T_{i j}^{2}\right) / \sum_{n n} \sum_{i j} \leq 3, \quad \bar{X} \quad$ is average transfer times, $T_{i j}^{0}, T_{i j}^{1}, T_{i j}^{2}$ from $\mathrm{i}$ to $\mathrm{j}$, respectively, the direct number of a transfer number, the number of secondary and two or more transfer. $d_{i j}$ is the traffic node $\mathrm{i}$ to $\mathrm{j}$ number of the total travel. Line length indicator $l_{\min } \leq l \leq l_{\max }$, usually $l_{\min }=5 \mathrm{~km}, l_{\max }=15 \mathrm{~km}$.

Importance such as by adjusting the node, select the maximum throughput of node $1 \mathrm{OD}$, and node a few lines already connected with a pack of all the OD flows, a few lines in the OD flow contains the largest selection and adjust a line extension of this road the other OD indicators.

Obtained after the adjustment point by point as amended by one of the public transportation network. Transit passengers at this time and then calculate the total travel time to the minimum core of the objective function: $\min T=\sum_{i=1}^{n} \sum_{j=1}^{n} a_{i j} T_{i j}$

and direct passengers to the largest rate $\max V=\sum_{i} \sum_{j} s_{i j} x_{i j} / \sum_{i} \sum_{j} t_{i j}$

of objective function $i j$. Function value obtained with the initial line of network function value compared to the results to see if satisfied with the adjustment.

\section{EXAMPLE:}

After planning OD matrix

TABLE II. AFTER PLANNING OD MATRIX

\begin{tabular}{|c|c|r|r|r|r|r|r|r|}
\hline \multicolumn{2}{|c|}{$\mathbf{1}$} & $\mathbf{2}$ & $\mathbf{3}$ & $\mathbf{4}$ & $\mathbf{5}$ & $\mathbf{6}$ & $\mathbf{7}$ & $\Sigma$ \\
\hline 1 & 33 & 56 & 124 & 51 & 150 & 111 & 280 & 805 \\
\hline 2 & 35 & 31 & 105 & 178 & 89 & 202 & 458 & 1098 \\
\hline 3 & 95 & 190 & 52 & 50 & 88 & 130 & 110 & 715 \\
\hline 4 & 90 & 14 & 73 & 23 & 173 & 219 & 303 & 895 \\
\hline 5 & 162 & 126 & 200 & 94 & 44 & 150 & 94 & 870 \\
\hline 6 & 136 & 89 & 214 & 231 & 149 & 51 & 205 & 1075 \\
\hline 7 & 259 & 121 & 237 & 269 & 239 & 268 & 37 & 1430 \\
\hline
\end{tabular}




\begin{tabular}{|c|l|l|l|l|l|l|l|l|}
\hline$\Sigma$ & 810 & 627 & 1005 & 896 & 932 & 1131 & 1487 & 6888 \\
\hline
\end{tabular}

Select the amount of matrix in the maximum OD 458, corresponding to the starting point for the 2 and finish at 7 , from 2 to 7 are 2-3-7,2-3-6-7,2-4-3-7 feasible path, 2-4-6-7, ... with Dijkstra's shortest path algorithm can be obtained for the 2-3-7. The initial line of this line into the net. And then removed 458, and then select from the matrix at this time the maximum 303 points always correspond to 4 and 7. From 4 to 7 are 4-3-7,4-6-7 feasible path, the shortest ... in comparison, the first with a 3-7 repeat the path length to be multiplied by the coefficient of 1.25 , making comparison, the the shortest path is $4-3-7$, the initial line of this line into the net.

OD matrix and then remove the amount of 303 , then select the 280, the corresponding point is always 1 and 7 , the calculated path is 1-5-7 after the election too, although the actual distance is longer than the path 1-3-7, but the 3 - path coefficient of 1.56 multiplied by 7 , obtained by repeating the path 1-5-7 than 1-3-7, the lines selected for the initial route network.

In turn can be regarded as 1-5-7,1-5-3-6,1-2-4,2-3-7,4-3-7,4-6-7 selected lines to form the initial line network.

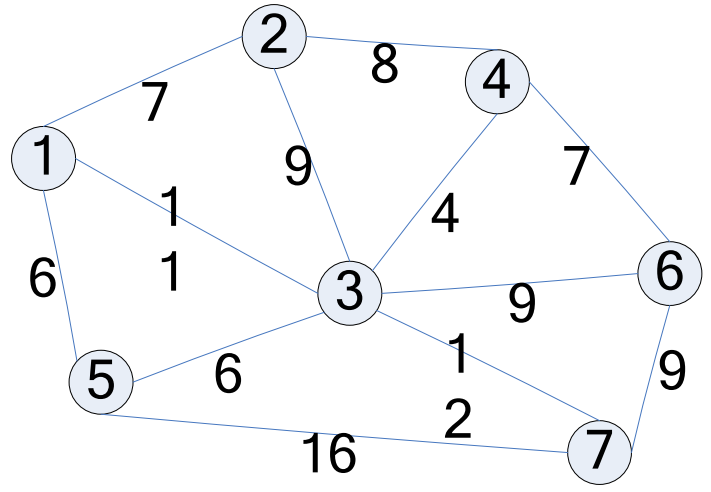

Figure 3. the initial line network

Then the last line in the OD matrix and the last column selected and the largest value of 1487 , corresponding to nodes 7, starting from node 7 amended 7 of the corresponding path to the calculation of the OD volume 7 , 2-3-7 and the path capacity and 568 OD path, 1-5-7 OD OD volume and volume and 413,4-6-7 374,4-3-7 path quantity and path of OD 508. After comparing the amount from the OD and the largest 2-3-7 line began to adjust. Adjustment and then select the second important transport node, followed point by point this way inspection and adjustment. Line network has been optimized for the final 1-5-7,1-5-3-6,1-2-4,2-3-7,4-3-7,2-4-6-7, satisfactory solution.

Results from the optimization process and the use of this algorithm can be seen from the form reflects the adjustment and optimization of each step to adjust the local feedback results. Because the core targeted in the initial line network optimization greatly reduces the amount of calculation and because the beginning of the line of thinking has to avoid the optimization of the local bad solution, making the process of wire mesh has been generated to ensure that the local optimum, and keep close to the optimal solution. And the initial line network has a more satisfactory solution, then the secondary goal of public transport needs to test adjustments, changes will not be too general, such as the test case, the 4-6-7 line, only to be adjusted to 2-4 -6-7 lines, more optimal understanding of the results. 2.1 compared to that of the optimization algorithm, this method is feasible, the optimization time is short, resulting in the deviation of the results will not be far off the local optimal solution, nor will there be other complex algorithms appear in the calculation of "combined explosion. "

The results of the optimization algorithm, the public transportation network in the actual planning of the social environment needs to take into consideration some factors such as road conditions, the station conditions, peak load capacity of the road, vehicle conditions, the state and city transportation policy. But this article focuses on the optimization algorithm, in order to facilitate the description, it does not take into account the above factors, but the actual planning of the constraints to be considered in order to ensure realistic planning programs.

\section{$\mathrm{V}$ CONCLUSION}

Bus problems are very complex, multi-objective public transport system, multi-factor, multi-functional and dynamic features of random problems to set up such a great challenge. In this paper, transit-oriented strategy, to study the OD matrix based public transportation network optimization problem, a optimized one by one into the net, and then gradually improved heuristic algorithm. Holds many lessons for a variety of response compared to NP-hard optimization problem, this algorithm is more simple and effective, easy to adjust.

\section{REFERENCES}

[1] Guowu zhang. Transport System. Chengdu: Southwest Jiaotong University Press, 1991.

[2] Wei Wang, Jiqian Xu. Urban transportation planning theory and method [M]. Beijing: China Communications Press, 1992.

[3] Weibin $\mathrm{Li}$, Cheng Xu. Introduction to urban public transport services engineering, Changchun: Jilin Science and Technology Press, 1994.

[4] Canqi Liu. Modern transportation planning study. China Communications Press, 2000.

[5] Wei Wang. Urban Transportation Planning Framework for Sustainable Development [J]. Southeast University, 2001, VoL31 (3) : $1-6$.

[6] Wei Wang, Xinmiao Yang, Xuewu Chen. Urban public transport system planning and manage technology. Beijing: Science Press, 2002.

[7] Shumin Feng, Hongren Chen. Public transit network optimization. Harbin Institute of Technology University, 2005, VoL37 (5) :691-693.

[8] Haode Liu, Xiaoguang Yang. Public Transit Network Optimization Based on Route Optimization Design Method. Computer and Communications .2007.

[9] Haode Liu, Xiaoguang Yang. Public Transit Network Based on Genetic Algorithm Optimization Design, Computer Engineering and Applications [J] .2007, VoL43 (8): 10 a 14

[10] Shengrui Zhang, Sea Yan. Urban Public Transportation Planning Theory and Practice [M] Beijing: China Railway Press, 2007.

[11] Zhaokun Kong. A rapid transit network optimization and evaluation methods [D] Dalian University of Technology, 2008. 
[12] Handsome Dai. Metropolitan public transport integration of key technologies [D]. Beijing: Beijing University of Civil Engineering, 2008.

[13] Kaiqiao Hu. Evaluation of Public Transit Road \& Automotive [D], 2009 (6).

[14] Zhenbao Wang,Yan-Yan Chen, Yuehua Zhu. Multi-level public transportation network planning method. Beijing University of Technology, 2011, VoL37 (2) :232-236.

[15] Yuan Zhou, Wei Deng,Qizhu Hu. Tabu algorithm based on genetic optimization of urban public transportation network. Wuhan University of Technology, 2011, VoL35 (2) 32-35.

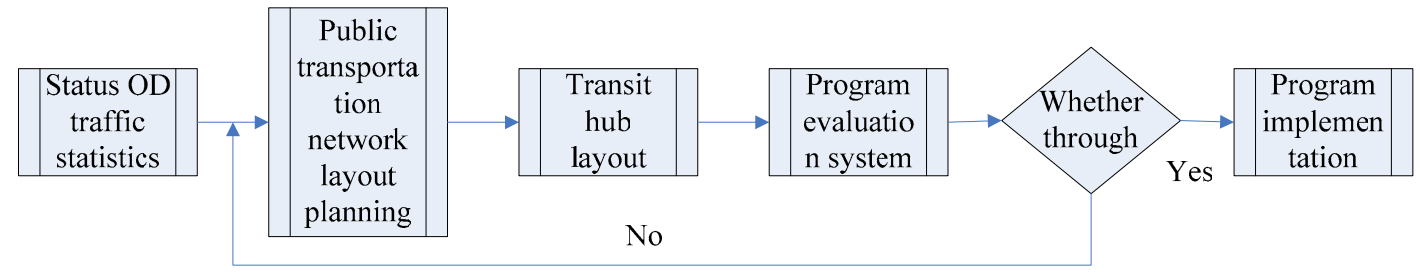

Adaptive public transportation network planning process

Figure 1.Adaptive public transportation network planning process

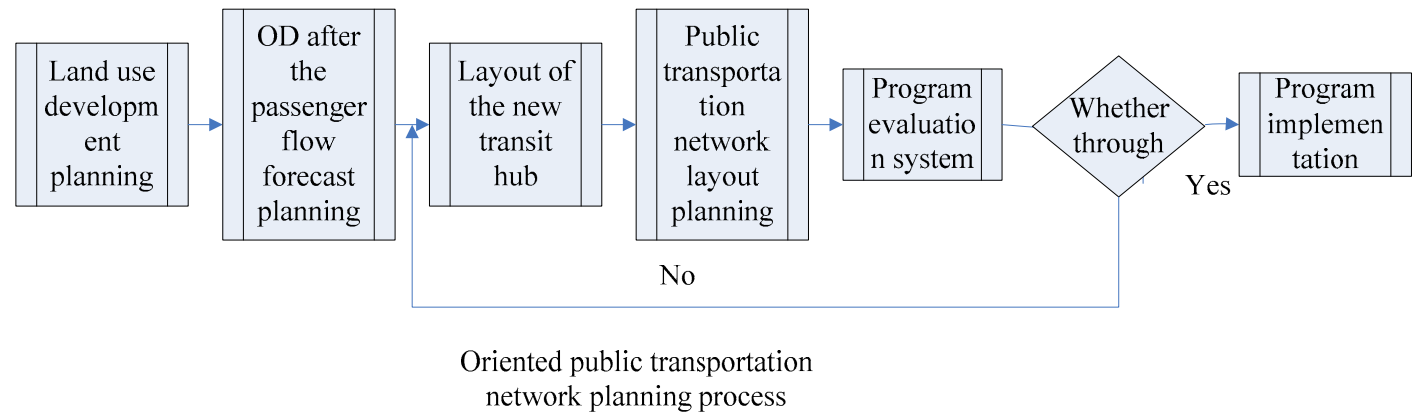

Figure 2. Oriented public transportation network planning process

TABLE I. PROPOSED DOUBLE-TRACK THE VALUE OF COEFFICIENT K

\begin{tabular}{|c|c|c|c|c|c|c|c|}
\hline Number of repeat lines & $\mathbf{0}$ & $\mathbf{1}$ & $\mathbf{2}$ & $\mathbf{3}$ & $\mathbf{4}$ & $\mathbf{5}$ & $\mathbf{6}$ \\
\hline Coefficient of repeat lines & 1 & 1.25 & 1.56 & 1.95 & 2.44 & 3.01 & 3.81 \\
\hline
\end{tabular}

